Revista de
Economíd
Contemporâned

\title{
DESEMPREGO E QUALIFICAÇÃO DA MÃO DE OBRA NO BRASIL
}

\author{
João Paulo Faria de Araújo ${ }^{a}$ \\ Mariangela Furlan Antigo ${ }^{b}$ \\ ${ }^{a}$ Graduado em Economia pela Universidade Federal de Minas Gerais (UFMG). \\ bProfessora Adjunta da Faculdade de Ciências Econômicas da UFMG (FACE/UFMG).
}

Artigo recebido em 18/12/2013 e aprovado em 15/10/2015.

RESUMO: Baseado no cenário do mercado de trabalho brasileiro na última década e inspirado pelas teorias da Busca do Emprego e do Capital Humano, o artigo em questão objetiva analisar o desemprego no Brasil através da perspectiva da qualificação da mão de obra entre 2002 e 2011, com base nos dados da Pesquisa Mensal de Emprego (IBGE). Os resultados se derivam de uma análise dinâmica que permite interpretar as mudanças no mercado de trabalho com base nas probabilidades de um indivíduo transitar entre as condições de emprego, desemprego e inatividade, e representam importante contribuição ao avanço da literatura sobre o tema, já que a maior parte dos estudos existentes apresenta uma análise de corte transversal de dados originados da Pesquisa Nacional por Amostra de Domicílios (IBGE), apontando apenas para taxas de desemprego estáticas. Para isso, utilizaram-se dois métodos de análise: um estudo univariado de matrizes de transição para cada um dos aspectos microeconômicos considerados e uma análise econométrica multivariada, por meio de um modelo Logit Multinomial. Dentre os resultados principais, constatou-se que as variáveis sexo, condição no domicílio, idade e escolaridade têm grande influência nas chances de um indivíduo conseguir emprego. Levando em consideração a qualificação do trabalhador, os resultados mostram que quanto mais escolarizado, menor o efeito adverso das

Correspondência para: João Paulo Faria de Araújo.

Contato: joaop8@gmail.com. 
características pessoais de um indivíduo, e que o grupo dos semiqualificados (aqueles que tem entre 4 e 10 anos de estudo) são os mais sensíveis a tais efeitos no sucesso pela busca do emprego. Por fim, o estudo mostra que os trabalhadores menos qualificados foram os que mais se beneficiaram com a mudança do cenário do mercado de trabalho brasileiro no período analisado.

PALAVRAS-CHAVE: mercado de trabalho; busca do emprego; capital humano; qualificação; análise dinâmica.

CLASSIFICAÇÃO JEL: J60; J63; J64. 


\title{
UNEMPLOYMENT AND WORKFORCE SKILL LEVELS IN BRAZIL
}

\begin{abstract}
Inspired by the theories of the Job Search and the Human Capital, this paper investigates the Brazilian job market with a view to analyze unemployment in Brazil between 2002 and 2011. It focuses the workforce skill levels and is based on data from the monthly employment survey published by IBGE. A dynamic analysis was conducted, which allowed interpreting the changes in the labor market based on the probability of an individual to transition among the conditions of employment, unemployment and inactivity. Two methods were employed in this investigation: a univariate study of transition matrices, which were applied to each of the microeconomics aspects considered and a multivariate econometrics analysis derived from a Multinomial Logit model. Several important results were found. Primarily, variables such as sex, role in the family, age and education have been found to have a significant influence on one's success in job search. The results reveal that the higher the level of education a worker has, the lower the influence of the aforementioned variables on the success of this worker's job search. Furthermore, those workers considered semi-qualified are the most sensitive to the influence of said traits on their chances of employment. Finally, the study shows that individuals in the group considered less qualified benefited the most from the changes in the Brazilian job market during the period under study. The results give an important contribution to the advancement of the literature on the subject, since most of the existing studies use a cross-sectional analysis of national household surveys, pointing only to static unemployment rates.
\end{abstract}

KEYWORDS: job market; job search; human capital; qualification; dynamic analyses. 


\section{INTRODUÇÃO}

A economia brasileira, desde 2002, tem evoluído de maneira significativa, o que gerou consequências muito positivas para o mercado de trabalho. Esse período foi marcado não só pelo aumento do crescimento do Produto Interno Bruto, mas também por inflação controlada a baixos níveis (se comparada ao histórico brasileiro), melhora na condição da balança comercial, crescimento do salário mínimo e geração de emprego muito acima do que tinha acontecido nos períodos anteriores, acompanhado de maior formalização da mão de obra.

De acordo com os dados do Instituto Brasileiro de Geografia e Estatística (IBGE), a taxa de desocupação das pessoas de dez ou mais anos de idade saiu de $12,9 \%$ em março de 2002 e chegou a 4,7\% em dezembro de 2011. Os dados chamam a atenção especialmente porque se percebe que a taxa de desocupação no Brasil não aparenta ter sofrido influência da crise econômica de 2008, já que, enquanto as principais economias do mundo como os Estados Unidos têm apresentado sérios problemas com elevação do desemprego e baixa criação de emprego, a taxa de desocupação no Brasil continuou caindo após 2008 e encontra-se muito abaixo do que era no período pré-crise.

A queda do desemprego foi acompanhada também por uma melhoria nas condições de trabalho e por um aumento do rendimento do trabalho médio em relação aos anos anteriores. Como mostra Saboia et al. (2009), a década de 1990 havia apresentado características de deterioração do trabalho no país. De acordo com os dados do Cadastro Geral de Emprego e Desemprego (CAGED) do Ministério do Trabalho (MTE), nesse período, o setor informal da economia cresceu significativamente. Além disso, houve aumento dos empregados sem carteira assinada e de trabalhadores por conta própria, assim como diminuição da remuneração média e baixo nível de geração de emprego. Já na década seguinte e, principalmente após 2004, houve um crescimento dos empregos com carteira assinada e o volume de empregos gerados saiu de uma média de 663 mil por ano entre 2000 e 2003 para uma média de 1.415 mil entre 2004 e 2009.

Ao considerar a questão da qualidade da mão de obra, dados do Instituto de Pesquisa Econômica Aplicada (IPEA, 2009) mostram que a proporção de desempregados com alta escolaridade comparada ao número total de desempregados no Brasil metropolitano cresceu muito na última década em relação às demais faixas de qualificação. O Gráfico 1 revela que essa razão aumentou de 36\% para 56\% para indivíduos com 11 ou mais anos de estudo entre julho de 2002 e o mesmo período em 2009. O fato de indivíduos mais qualificados representarem a maior parte dos desempregados, e essa proporção ter aumentado na última década, pode ter como um dos seus principais motivos uma característica do mercado brasileiro apontada por Pochmann et al. (2007). Segundo o autor, existem no Brasil milhares de trabalhadores de alta qualidade que estão desempregados e muitos postos de trabalho para mão de obra qualificada 
não preenchidos, de forma que existe um desencontro entre a oferta e a demanda por trabalhadores de diferentes níveis de qualificação.

A proporção de desempregados do grupo de indivíduos que têm entre 4 e 10 anos de estudo em relação ao número total de desempregados saiu de 55\% em 2002 para $40 \%$ em 2009, permanecendo significativa. Camargo e Reis (2005) definem as pessoas nessa faixa de escolaridade como semiqualificados e argumentam que um dos seus principais problemas na busca pelo emprego é a questão da assimetria da informação. Segundo os autores, os semiqualificados apresentam heterogeneidade muito grande em sua composição, gerando dificuldade na percepção dos empregadores em relação à qualidade desses indivíduos e, consequentemente, afetando a contratação dos mesmos.

O Gráfico 1 mostra, ainda, que a proporção de trabalhadores com 0 a 3 anos de anos de estudo caiu 60\% entre 2002 e 2009, chegando a pouco menos que $4 \%$ da quantidade total de desempregados, o que mostra que trabalhadores com baixa escolaridade tem se beneficiado de maneira significativa com a queda da taxa de desemprego no Brasil. Saboia et al. (2009) corroboram essa tendência e uma de suas constatações é a forte geração de empregos para aquelas pessoas com baixo nível de qualificação.

\section{Gráfico 1 - Brasil metropolitano - evolução da composição dos desempregados segundo faixa de escolaridade}

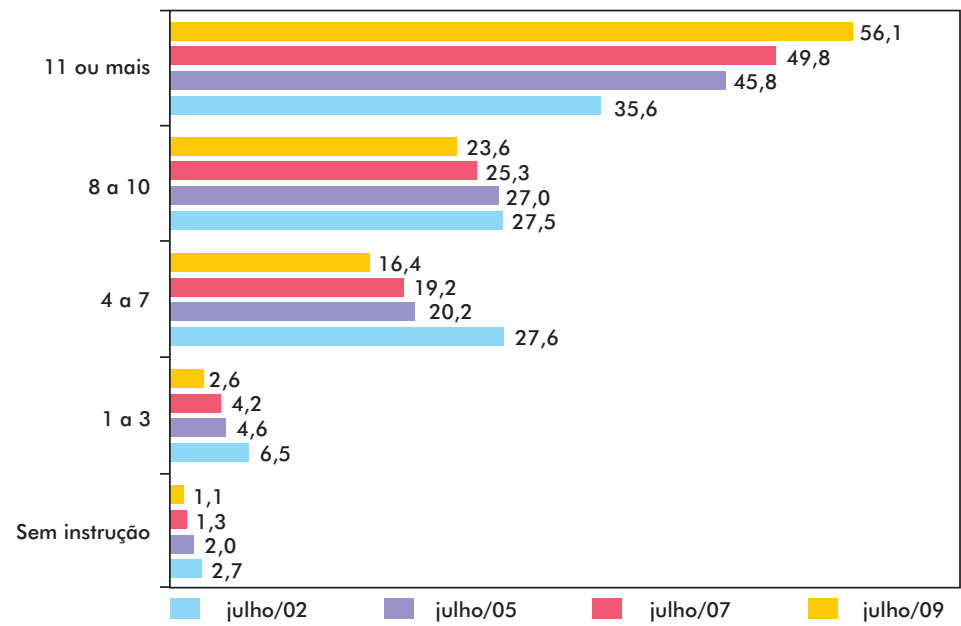

Fonte: Elaborado por IPEA (2009), com base em dados de IBGE (2009).

A literatura sobre o desemprego aponta que tais diferenças na taxa de desocupação para os diferentes níveis de qualificação e outras características do indivíduo não são um fenômeno particular do mercado brasileiro. Layard, Nickell e Jackman (1991), por exemplo, enfatizam que essa tendência é presente em vários países e acontece de forma muito parecida entre eles: 
As everybody knows, unemployment rates differ widely between occupations and between regions, as well as across age, race, and (sometimes) sex groups. The striking thing is how stable these differences are. In all countries, unskilled people have much higher unemployment rates than those with skills. Similarly, youths have higher rates than adults. (LAYARD, NICKELL e JACKMAN, 1991, p. 285)

Baseado no cenário do mercado de trabalho brasileiro na última década e balizado pelas teorias da busca do emprego e do investimento em capital humano, o presente artigo objetiva analisar o desemprego no Brasil através da perspectiva da qualificação da mão de obra. É importante ressaltar que esse estudo representa importante contribuição à literatura existente ao realizar uma análise longitudinal dos dados da Pesquisa Mensal de Emprego (PME), o que implica resultados mais detalhados da evolução da probabilidade de desemprego para os grupos apresentados. Enquanto a maior parte dos estudos existentes apresenta uma análise de corte transversal de dados originados da Pesquisa Nacional por Amostra de Domicílios (PNAD), apontando apenas para as taxas de desemprego estáticas, o presente artigo busca contribuir no sentido de que, ao se basear em dados longitudinais, interpretar o mercado de trabalho de forma dinâmica e discutir probabilidades de transição entre as condições de emprego, desemprego e inatividade. Mais especificamente, o trabalho busca: verificar quem mais se beneficiou com a queda da taxa de desemprego do Brasil entre 2002 e 2011 de acordo com a escolaridade; analisar os fluxos entre os estados do mercado de trabalho segundo os atributos pessoais dos indivíduos; e apontar, para os diferentes níveis de qualificação da mão de obra, quais variáveis têm maior influência na probabilidade de um indivíduo encontrar ocupação (ou manter-se desempregado). Para tanto, o artigo está dividido em cinco seções, incluindo a introdução. A próxima seção apresenta as evidências teóricas e empíricas acerca do assunto. A base de dados e as metodologias utilizadas são apresentadas na terceira seção. Segue-se, na seção seguinte, com a apresentação e a discussão dos resultados e, por fim, são apresentadas as conclusões gerais.

\section{DESEMPREGO: EVIDÊNCIAS TEÓRICAS E EMPÍRICAS}

\subsection{TEORIA DA BUSCA DE EMPREGO E DO INVESTIMENTO EM CAPITAL HUMANO}

O desemprego é um fenômeno muito estudado devido à sua importância para a economia de um país, pois tanto a diminuição de novos empregos gerados como a demissão de indivíduos empregados, comumente, desencadeiam outros problemas para a sociedade. No nível macro, destacam-se problemas que vão do aumento do índice de 
criminalidade, pobreza e número de desabrigados, ao aumento da taxa de mortalidade (além do aumento do índice de suicídio) e doenças cardiovasculares. Em relação à economia, um maior índice de desocupação leva, por exemplo, a maiores gastos por parte do governo com seguro desemprego, sem contar com o custo de oportunidade da renda que aqueles desempregados estariam gerando para a economia do país se eles tivessem um salário e estivessem consumindo bens e serviços. No nível micro, o desemprego leva, em muitos casos, a problemas de estresse, depressão e baixa autoestima. Além disso, uma das suas piores consequências para o indivíduo é a perda de conhecimento e habilidades previamente adquiridas, levando a um desinvestimento em capital humano.

Após a crise econômica de 2008, muitos países como Estados Unidos, Espanha e Portugal vêm sofrendo com altas taxas de desocupação e enfrentando dificuldade para reverter tal cenário, o que é preocupante devido às potenciais consequências do desemprego discutidas no parágrafo anterior. Já outros países como o Brasil, Alemanha e Áustria chegaram a apresentar melhora na taxa de desemprego após 2008, o que aponta para a heterogeneidade do efeito da crise em diferentes países. Além disso, é importante perceber que, em um mesmo país, as mudanças no cenário de desemprego afetam diferentes grupos da população de forma muito diferente, como é o caso do Brasil (este trabalho mostrará que os indivíduos com menos escolaridade se beneficiaram consideravelmente mais do que indivíduos com mais escolaridade nos últimos dez anos em temos de probabilidade de sucesso na busca de emprego). Tais discrepâncias levam economistas e outros estudiosos a indagar quais são os fatores que realmente determinam ou influenciam as mudanças no nível de desemprego.

A maior parte dos estudos realizados a respeito do tema aponta para a explicação do desemprego através da análise de fatores macroeconômicos como, por exemplo, crescimento do PIB do país, intensificação do progresso tecnológico e mudanças na taxa de inflação. O presente trabalho busca, no entanto, aprofundar a análise do desemprego através de uma perspectiva microeconômica, de forma a entender as características do indivíduo que influenciam a taxa de desemprego e por que diferentes grupos de indivíduos no mesmo país são afetados de forma tão desigual.

O modelo de busca de emprego, baseado em Layard, Nickell e Jackman (1991), mostra que a questão do desemprego é complexa e afetada por muitos fatores, além daqueles macroeconômicos e conjunturais. Nesse sentido, os autores fornecem uma contribuição muito importante para a investigação do desemprego e para o entendimento de como se dá sua dinâmica através da análise de suas causas. Os autores questionam se a atitude dos desempregados na busca do emprego é um fator importante e, por exemplo, se as características dessas pessoas que estão procurando emprego é o que determinará seu sucesso na busca. Com isso, a grande contribuição dos 
autores para a teoria do desemprego é a busca pela explicação desse fenômeno por meio de atributos pessoais daqueles que estão no mercado de trabalho procurando ocupação.

A afirmação básica do modelo de acordo com Layard, Nickell e Jackman (1991) é de que um número enorme de fatores afeta o desemprego através de uma variável definida como "efetividade da busca do desempregado", e não somente o fazem as pressões salariais dos sindicatos, resistência por parte dos trabalhadores baseadas no salário real e medidas como seguro-desemprego. Esses autores incluem dentro da efetividade da busca do desempregado tudo que explique e/ou exerça influência na velocidade na qual aquele indivíduo consegue uma ocupação, como por exemplo: a eficiência na qual a informação sobre vagas são transmitidas, o esforço e o tempo gasto pelo trabalhador para procurar emprego, a prática de recrutamento das empresas, a legislação de proteção ao trabalhador que deixa os empregadores mais cautelosos na contratação, a duração do período ao longo do qual a pessoa fica desocupada e, principalmente, como as características individuais dos desempregados atendem às necessidades das vagas oferecidas.

O modelo de busca por emprego baseia-se na função de contratação $H=h(V, c U)$, em que o número de contratação $(H)$, que é identificado pelo número de pessoas que deixam o desemprego, depende do número de vagas $(V)$ e do número de pessoas que, efetivamente, procuraram emprego $(c U)$, sendo $U$ o número de pessoas desempregadas e $c$ a efetividade média de busca.

O modelo considera que toda contratação envolve o casamento de uma vaga com uma pessoa que está procurando emprego, de forma que a função $h($.) é linearmente homogênea em $V$ e $c U$, isto é, o número de contratações dobra se o número de indivíduos que efetivamente procura emprego dobra. Dividindo-se ambos os lados por $c U$, tem-se: $H / U=c_{i} h(V / c U, 1)$, onde $c_{i}=c\left(B_{i} / \mu_{w}, q_{i}\right)$ e $B_{i}$ representa a renda do indivíduo; $\mu_{w}$ o salário médio que o trabalhador espera ganhar e, consequentemente, $B_{i} / \mu_{w}$, uma medida do rendimento relativo. A variável $q_{i}$ da função de $c_{i}$ representa os atributos pessoais do indivíduo que está buscando o emprego, como sexo, cor, idade e escolaridade, os quais, juntamente com o rendimento relativo, representam as características individuais. O outro fator são as variáveis macroeconômicas, que são fatores conjunturais refletidos pelo grau de competição por vagas.

Desta maneira, através do modelo apresentado, é possível desagregar a taxa de desemprego de acordo com as características individuais e comportamento macroeconômico. Dentro dos atributos individuais, merece destaque o papel da escolaridade. A escolaridade, vista como investimento em capital humano, pode afetar positivamente a probabilidade de o indivíduo encontrar um emprego e apresenta correlação positiva com a remuneração média do trabalhador. 
Becker (1975) afirma que investimentos em educação, escolarização, treinamentos e cursos são considerados investimentos em capital humano, pois aumentam o ganho do indivíduo ao longo da vida. Assim, o contínuo crescimento da renda per capita de muitos países durante o século XIX e XX se deu pela expansão do conhecimento científico e técnico que aumenta a produtividade do trabalho. A teoria ainda alerta para o fato de que novos avanços tecnológicos têm pouco valor se poucos trabalhadores qualificados existem no país para tirar vantagens daquela tecnologia, de forma que crescimento econômico (e, consequentemente, melhoria nas condições de desemprego) depende de uma relação muito próxima das sinergias entre conhecimento e capital humano com o advento de novas tecnologias. Japão, Taiwan, China e outros países asiáticos apresentam-se como bons exemplos dessa combinação. Tais países são marcados por rápido crescimento econômico e as características de mão de obra qualificada e bem treinada podem ser apontadas como a principal causa desse triunfo.

\subsection{REVISÃO DA LITERATURA}

O problema do desemprego nas últimas décadas do século passado apresentava-se como uma das maiores preocupações dos brasileiros devido ao alto nível de taxa de desocupação e alta discriminação das minorias no mercado de trabalho. Com isso, vários estudos foram realizados acerca do assunto durante aquele período, bem como nos primeiros anos da década passada. A presente seção apresenta as principais literaturas empíricas e seus principais resultados acerca do tema, focando nas informações pertinentes ao objetivo do trabalho e nos modelos teóricos discutidos na seção anterior.

Sampaio (2012) analisa para a Região Sul do Brasil as transições que ocorreram no mercado de trabalho entre empregados, desempregados e aqueles que estavam fora do mercado de trabalho entre 1995 e 2005 . O autor investiga como variáveis macroeconômicas (como a taxa de mortalidade, os choques tecnológicos e os choque externos) e fatores microeconômicos (como idade, escolaridade, sexo, condição na família e região geográfica) podem influenciar esses fluxos para pessoas com idade entre 16 e 70 anos. Para a realização do estudo, foram utilizados os dados da PNAD e o modelo econométrico considerado foi o Logit Multinomial. As análises realizadas mostram que os homens têm maior probabilidade de estar ocupados e menor probabilidade de estar fora do mercado de trabalho. Além disso, o estudo mostra uma relação positiva entre idade e probabilidade de estar empregado, com evidência de um ponto máximo, aproximadamente, aos 40 anos.

Fernandes, Lima e Santos (2008) apontam que a taxa de desemprego atinge de maneira desigual diferentes grupos sociais ou diferentes segmentos da força de traba- 
lho. Usando a PNAD de 2005 e um Logit Multinomial, os autores mostram que as taxas de desemprego são mais elevadas para mulheres de até 24 anos do que para aquelas que se encontram nas demais faixas etárias. Além disso, mostram que fatores como escolaridade, renda, experiência e cor são atributos que levam o indivíduo a ser afetado diferentemente pelas taxas de desemprego, além de considerarem também a variável maternidade, já que o foco do estudo é o público feminino. Os resultados encontrados apontam para uma diminuição de probabilidade de desemprego para mulheres com mais escolaridade.

Com uma abordagem semelhante, Mendonça, Lima, Lírio (2009) estudam o mercado de trabalho para as mulheres entre 15 e 24 anos no nordeste brasileiro, baseados nos dados da PNAD de 2006 por meio da estimação de um Logit Multinomial e os resultados mostram que experiência, escolaridade, renda e existência de filho são as variáveis explicativas mais importantes do modelo. Quanto ao desemprego das mulheres jovens, vale também citar Santos, Leal e Fernandes (2010), que realizam um estudo muito parecido, mas para o ano de 2008 em Minas Gerais, e também apontam que as mulheres jovens até 24 anos apresentam maior desemprego do que as demais faixas etárias e do que os homens da mesma idade.

Cunha, Araújo e Lima (2011) também usam dados da PNAD e um Logit Multinomial para analisar os atributos individuais na faixa etária entre 16 e 29 anos no ano de 2007. Apontam que jovens do sexo feminino, não brancos, com menor grau de escolaridade e que ocupam posição de cônjuge na unidade familiar são mais propícios a se encontrar na posição de inativos ou desempregados.

Camargo e Reis (2007) analisam o mercado de trabalho para os jovens brasileiros por meio dos efeitos da estabilização da inflação no Brasil após 1994, com a implementação do Plano Real. Através de testes empíricos baseados nos dados da PNAD de 1981 a 2002, os autores concluem que as reduções nas taxas de inflação no país aumentaram a taxa de desemprego e que os mais afetados por tal cenário foram os jovens entre $18 \mathrm{e}$ 20 anos. Fernandes e Picchetti (1999), com base na PNAD de 1995 e o uso do modelo Logit Multinomial, mostram que a probabilidade de desemprego atinge seu máximo para trabalhadores com nove anos de estudo e mostram que a relação idade e inatividade é de alta inatividade para os mais jovens e mais velhos.

A questão da qualificação do trabalhador como variável determinante do desemprego é considerada por Camargo e Reis (2005). Os autores tratam a relação entre essas duas variáveis por meio de uma perspectiva diferente: a da assimetria da informação. Empiricamente, é constatado que a relação entre a taxa de desemprego brasileira e a qualificação do trabalhador tem um formato de U-invertido. Além disso, constatam que a taxa de desemprego para os jovens é muito maior do que a dos demais trabalhadores para diferentes faixas etárias e explicam tal tendência pela dificuldade de 
um empregador julgar a qualidade da mão de obra do jovem sem que ele tenha provado sua qualificação no mercado de trabalho. Assim como os jovens, os trabalhadores classificados como semiqualificados (entre 4 e 10 anos de estudo) também aparecem como os mais prejudicados pela assimetria da informação, já que por serem um grupo muito heterogêneo, os empregadores teriam dificuldade em inferir seus níveis de qualificação.

\section{BASE DE DADOS E METODOLOGIA}

\subsection{BASE DE DADOS}

A base de dados utilizada nesse trabalho foi a Pesquisa Mensal de Emprego (PME), realizada pelo Instituto Brasileiro de Geografia e Estatística (IBGE) durante o período de 2002 a 2011 ${ }^{1}$. A PME é uma pesquisa que tem como principal objetivo fornecer indicadores sobre o desempenho conjuntural do mercado de trabalho, principalmente relativo ao desemprego, sendo reformulada a partir de 2002, devido a mudanças na estrutura produtiva do Brasil, na relação de trabalho e na alocação da mão de obra. $\mathrm{O}$ levantamento amostral é feito para as seis regiões metropolitanas do Brasil, a saber: Recife, Salvador, Belo Horizonte, Rio de Janeiro, São Paulo e Porto Alegre. Umas das principais vantagens do banco de dados gerado por essa pesquisa é a possibilidade de se fazer o acompanhamento do indivíduo ao longo do tempo. Para tanto, a PME adota um sistema de rotação de painéis, o que quer dizer que, ao invés de os domicílios serem entrevistados durante todos os meses da pesquisa, existe um padrão definido para sua entrada e saída na amostra. Conhecido como padrão 4-8-4, o esquema de rotação da PME determina que de outubro de um ano ímpar a setembro do ano seguinte, todo mês, um grupo de domicílios entra na pesquisa e é entrevistado por quatro meses consecutivos. Após os quatro meses, o grupo sai da amostra e não é entrevistado durante os oito meses seguintes. O retorno do grupo acontece logo em seguida e são feitas mais quatro entrevistas, após as quais o grupo sai definitivamente da amostra. Com o intuito de minimizar o possível atrito do acompanhamento longitudinal do indivíduo, utilizamos nesse trabalho o algoritmo proposto por Ribas e Soares (2008).

1 O período escolhido permitiu alcançar as principais conclusões em relação ao tema estudado desde 2002 até a atualidade. As probabilidades de transição se mantiveram relativamente constantes durante o período analisado, inclusive entre 2008 e 2009, quando o país foi atingido pela crise internacional. Modificações nas probabilidades de transição podem vir a ocorrer no período mais recente, uma vez que os primeiros sinais de desaceleração do mercado de trabalho foram registrados em 2014. 
As proporções dos entrevistados da PME durante os anos de 2002 e 2011 para a primeira entrevista são apresentadas a seguir. A análise é restrita à população brasileira acima de 15 anos, excluindo os pensionistas, empregados domésticos e parentes dos empregados domésticos. Como o Gráfico 2 mostra, 54\% dos entrevistados na primeira entrevista durante os anos de 2002 a 2011 são mulheres. Para a classificação dos indivíduos em relação à cor, foram considerados dois grandes grupos: brancos e não brancos. O grupo de indivíduos da cor branca, composto por aqueles que responderam ser brancos ou amarelos, representa $44 \%$ das observações. O grupo não branco inclui os indivíduos que se declararam ser pretos, pardos ou indígenas. Quanto à condição no domicílio, verificou-se que $41 \%$ dos entrevistados eram chefes. Pelo Gráfico 5, podemos observar que a maior parte dos entrevistados se encontra na faixa etária entre 30 e 49 anos, seguido pelo grupo mais jovem (entre 15 e 29 anos).

Em relação à escolaridade, os entrevistados foram divididos em três grandes faixas. Foram classificados como tendo alta escolaridade (ou altamente qualificados) aqueles indivíduos com 11 ou mais anos de estudo, enquanto os que possuem entre quatro e dez anos de estudo foram classificados como semiqualificados e, por último, foram agrupados os indivíduos que tinham entre zero e três anos de estudo (baixa qualificação). O Gráfico 6 mostra que a maior parte dos entrevistados encontra-se nos grupos semiqualificados e altamente qualificados.
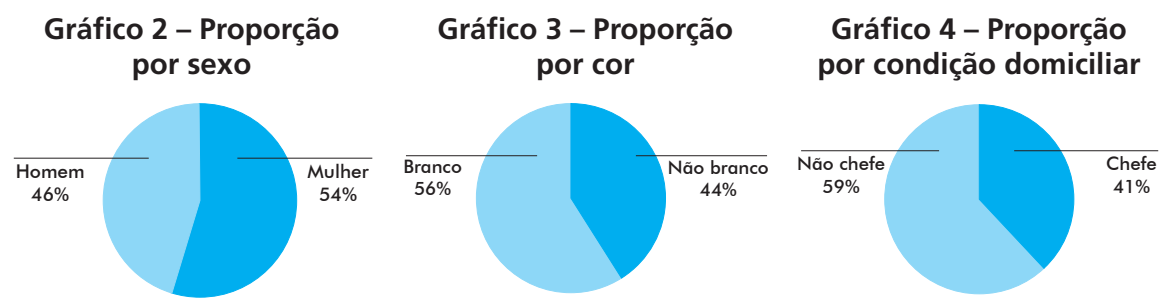

Fonte: Elaboração própria com base em dados de 2002 a 2011 da PME/IBGE.
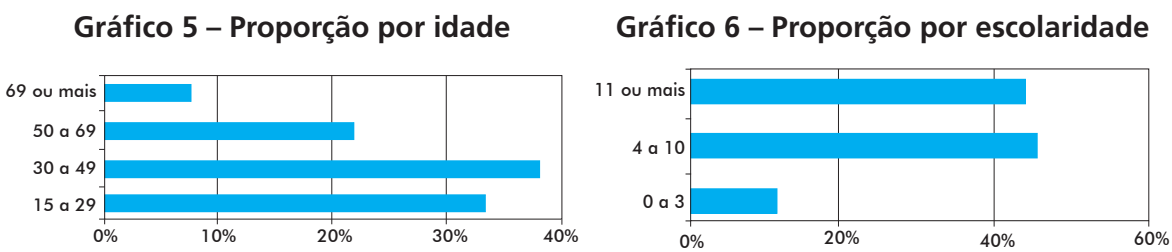

Fonte: Elaboração própria com base em dados de 2002 a 2011 da PME/IBGE. 


\subsection{MÉTODOS DE ANÁLISE}

Dois métodos de análise foram utilizados nesse artigo. O primeiro deles é uma análise univariada que irá se basear em um estudo descritivo de cada uma das variáveis microeconômicas estudadas: cor, sexo, condição no domicílio, idade, escolaridade e região metropolitana. Baseado no trabalho de Clark e Summers (1990), o comportamento do indivíduo segundo suas características individuais pode ser representado por uma matriz de probabilidade de transição $p^{\mathrm{i}}$. Essa matriz de transição, também conhecida como matriz estocástica ou matriz de Markov, será utilizada nesse estudo para representar a dinâmica de mobilidade dos indivíduos entre três estados no mercado de trabalho - empregado, desempregado e inativo. Sua representação é dada da seguinte maneira:

$$
p^{i}=\left(\begin{array}{ccc}
P_{e e}^{i} & P_{e d}^{i} & P_{e i}^{i} \\
P_{d e}^{i} & P_{d d}^{i} & P_{d i}^{i} \\
P_{i e}^{i} & P_{i d}^{i} & P_{i i}^{i}
\end{array}\right)
$$

Nela, $P_{l, k}^{i}$ representa a probabilidade de o indivíduo $i$ estar no estado $k$ (representada pelo subscrito "e" para empregado, "d" para desempregado e "i" para inativo) no período $t+1$, condicionado ao fato de ele se encontrar no estado $l$ no período $t$.

A segunda metodologia adotada baseia-se em uma análise econométrica multivariada, através de um modelo Logit Multinomial, presente em Wooldridge (2007) e Gujarati (1995) Com esse modelo, é possível entender como as mudanças no conjunto de variáveis explicativas $x$ (variáveis relativas a atributos pessoais dos entrevistados já apresentadas no parágrafo anterior) e $z$ (variáveis de ano que irão captar as variações macroeconômicas) afetam a probabilidade de um indivíduo encontrar-se empregado $(j=1)$, desempregado $(j=2)$ ou inativo $(j=3)$, dado que ele estava desempregado no período anterior $(k=2)$.

O Modelo Logit Multinomial é expresso como segue:

$$
P\left(y_{i t}=j \mid y_{i(t-1)}=k, x_{i}, z_{i}\right)=\exp \left(\beta_{0}+x_{m i t} \beta_{m}+z_{n i t} \beta_{n}\right) /\left[1+\sum^{j}{ }_{t=0} \exp \left(\beta_{0}+x_{m i t} \beta_{m}+z_{\text {nit }} \beta_{n)}\right]\right.
$$

No modelo, $j=1,2,3$ e $k=2$.

Para estimar esse modelo, usaremos o método da máxima verossimilhança através da função: 


$$
1_{i}(\beta)=\sum_{t=0}^{j} 1\left[y_{i}=j\right] \log \left[p_{j}\left(w_{i}, \beta\right) \text {, onde } w_{i}=\left(x_{i}, z_{i}\right)\right.
$$

Para interpretação do resultado, deve-se utilizar a seguinte razão:

$$
p_{j}(w, \beta) / p_{0}(w, \beta)=\exp \left(w \beta_{j}\right)
$$

Essa razão representa a relação entre as probabilidades de cada estado em relação ao estado de referência.

\section{ANÁLISE DE RESULTADOS}

\subsection{MATRIZES DE TRANSIÇÃO}

Os principais resultados das matrizes de transição são apresentados a seguir ${ }^{2}$. Será apresentado também o resultado estratificado por qualidade da mão de obra, considerando três faixas de escolaridade: i) mais qualificados: indivíduos com mais do que 11 anos de estudo; ii) semiqualificados: aqueles que têm entre 4 e 10 anos de estudo; e iii) menos qualificados: aqueles com 0 a 3 anos de estudo.

\section{Gráfico 7 - Probabilidade de se manter empregado segundo o sexo}

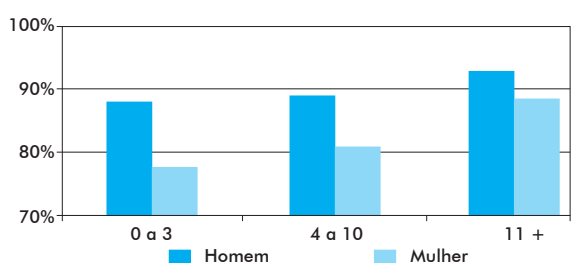

Gráfico 8 - Probabilidade de encontrar emprego dado desemprego segundo o sexo

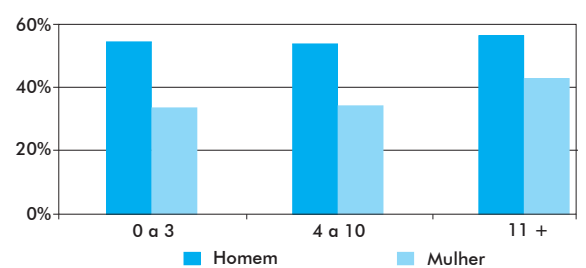

Fonte: Elaboração própria com base em dados de 2002 a 2011 da PME/IBGE.

Quanto ao sexo, percebe-se uma diferença significativa na probabilidade de indivíduos mudarem de status quanto às condições de emprego. A probabilidade de um homem permanecer empregado é consideravelmente maior. A diferença é ainda maior na probabilidade de um homem conseguir emprego na quinta entrevista dado o de-

\footnotetext{
2 As matrizes de transição completas estão disponíveis com os autores para quem as solicitar.
} 
semprego na primeira. Quanto à análise estratificada, os Gráficos 8 e 9 mostram que a dificuldade das mulheres no mercado de trabalho quando comparadas aos homens agrava-se para aquelas que têm menor nível de qualificação.

A questão de discriminação racial ainda representa um grande problema social brasileiro e sabemos que a renda média por trabalhador não branco é menor do que a para trabalhadores brancos. No entanto, a matriz de transição abaixo mostra que a dinâmica de mobilidade dos indivíduos entre os três estados no mercado de trabalho não muda muito baseado no atributo pessoal cor, tendo os brancos uma pequena vantagem na chance de encontrar emprego ou manter-se empregado. Da mesma forma que para a análise geral, a análise segundo a qualidade da mão de obra mostra que a diferença na probabilidade de manter-se empregado ou conseguir emprego não muda significativamente para a variável cor.

Gráfico 9 - Probabilidade de se manter empregado segundo a cor

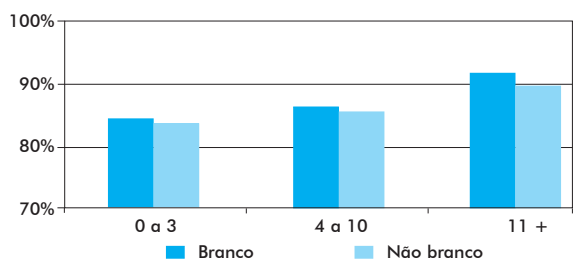

Gráfico 10 - Probabilidade de se encontrar emprego dado desemprego segundo a cor

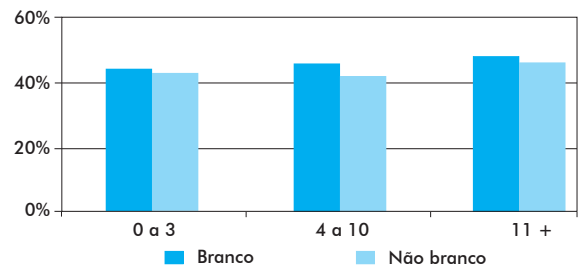

Fonte: Elaboração própria com base em dados de 2002 a 2011 da PME/IBGE

Devido à necessidade de sustentar os demais familiares, o comportamento dos chefes tende a ser diferenciado quando comparados aos não chefes. Geralmente, os indivíduos chefes tendem a procurar maior estabilidade no emprego, o que pode ser uma explicação para sua maior probabilidade de permanecer empregado de um ano para o outro. Além disso, os resultados mostram que a chance de um indivíduo sair da condição de desemprego para emprego é $11 \%$ maior para os chefes do que para os não chefes, o que levanta a hipótese de que o fato de ser chefe do domicílio afete positivamente a chance de um indivíduo conseguir emprego no mercado de trabalho brasileiro.

A análise da condição domiciliar estratificada por qualificação do trabalhador mostra que ser ou não ser chefe de família tem menor influência na probabilidade de um indivíduo qualificado encontrar emprego do que para aqueles indivíduos menos qualificados, como mostra o Gráfico 13. Além disso, o Gráfico 12 mostra que a diferença na probabilidade de manter-se empregado entre chefes e não chefes cresce para os semiqualificados e menos qualificados, o que sugere que a hipótese inicial de que chefes tendem a procurar maior estabilidade no emprego é mais significativa para fa- 
mílias menos abastadas, já que, em muitos desses casos, o chefe será a única fonte de renda da casa.

Gráfico 11 - Probabilidade de se manter empregado segundo a condição domiciliar

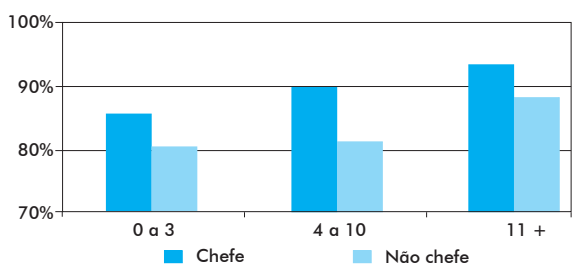

Gráfico 12 - Probabilidade de encontrar emprego dado desemprego segundo a condição domiciliar

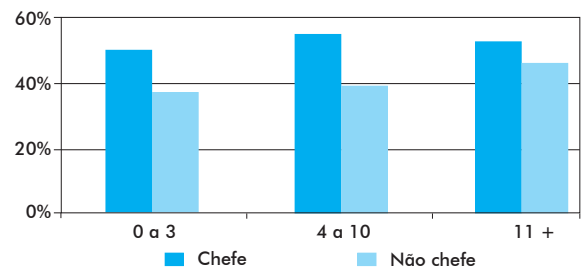

Fonte: Elaboração própria com base em dados de 2002 a 2011 da PME/IBGE.

Observa-se que o atributo pessoal idade é um fator que influencia significativamente as probabilidades de mudanças entre os estados do mercado de trabalho. A análise revela que indivíduos mais jovens (entre 15 e 29 anos) têm menor probabilidade de encontrar emprego ou se manterem empregados do que indivíduos com idade entre 30 e 49 anos. Ao analisar a probabilidade de indivíduos manterem-se empregados para os diferentes níveis de qualificação, pode-se concluir através do Gráfico 13 que aqueles com mais escolaridade têm maior probabilidade de se manterem empregados para todas as diferentes faixas etárias.

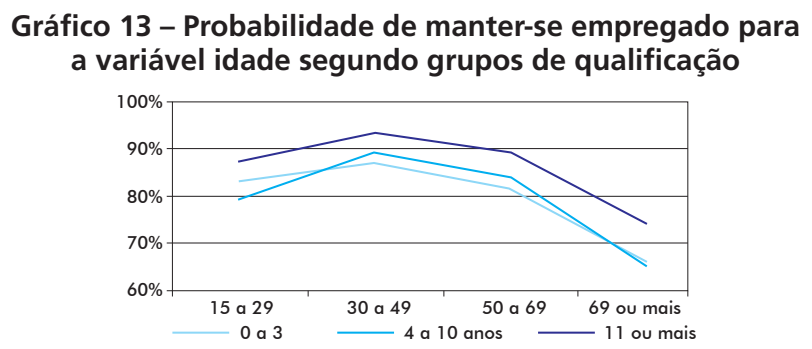

Fonte: Elaboração própria com base em dados de 2002 a 2011 da PME/IBGE.

Para a probabilidade de saírem da condição de desemprego para emprego, a tendência é a mesma apresentada anteriormente. Os indivíduos mais jovens têm menor probabilidade de encontrarem emprego quando estão na condição de desempregados do que os indivíduos que têm entre 30 e 49 anos. Considerando a questão da qualidade da mão de obra, os jovens mais qualificados sofrem menos com essa disparidade do que os menos qualificados, já que a diferença de probabilidade de tal ocorrência para eles é de apenas $1 \%$ contra $9 \%$ para os semiqualificados e $6 \%$ para os menos qualificados. 


\section{Gráfico 14 - Probabilidade de encontrar emprego para a variável idade segundo grupos de qualificação}

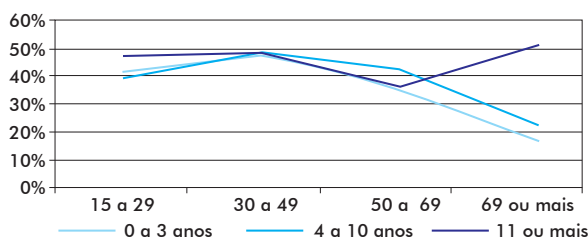

Fonte: Elaboração própria com base em dados de 2002 a 2011 da PME/IBGE.

A análise das tabelas de transição mostra que não houve muita diferença nas probabilidades de transição no mercado de trabalho para os anos entre 2002 e 2011. De certa forma, era esperado que houvesse uma mudança nessa dinâmica para os anos em que a crise financeira foi mais acentuada, já que empregadores estariam mais receosos na contratação de trabalhadores devido à incerteza dos rumos da economia. Não obstante, a análise das tabelas de transição de 2008 e 2009 mostra que isso não ocorreu. A probabilidade de um indivíduo continuar empregado no ano de 2008 foi de $88 \%$ e, para 2009, essa probabilidade foi de $90 \%$, até um pouco maior do que a média dos demais anos. Além disso, a probabilidade de um indivíduo que estava desempregado na primeira entrevista de 2008 ter conseguido emprego um ano depois foi tão alta quanto a dos demais anos. Com isso, podemos concluir que, entre os anos de 2002 e 2011, pode não ter havido diferença considerável nas probabilidades de mudança de status dos indivíduos devido ao fator período, o que aponta para o fato de que a crise mundial de 2008 não afetou o mercado de trabalho brasileiro de forma severa. O Gráfico 15 a seguir corrobora essa conclusão e mostra que, apesar de um leve aumento no desemprego entre os meses de janeiro e abril de 2009, a tendência continuou sendo de queda durante e após a crise financeira.

\section{Gráfico 15 - Taxa de desocupação nas regiões metropolitanas do Brasil}

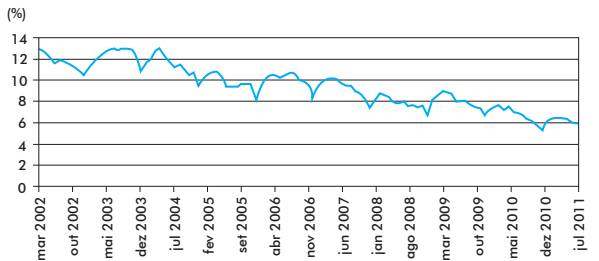

Nota: Consideradas as pessoas com mais de dez anos de idade. Fonte: Elaboração própria com base em dados da PME/BGGE. 
Como foi visto, o estudo detalhado das variáveis de atributo individual em relação à probabilidade de sucesso na busca de ocupação sugere que condição no domicílio, sexo, idade e escolaridade têm grande influência nas chances de um indivíduo conseguir emprego. Concluiu-se que ser chefe, ser homem, ter idade entre 30 e 49 anos, ou ter 11 ou mais anos de estudo são características que aumentam a probabilidade de conseguir emprego ou manter-se empregado quando comparado às pessoas que não possuem tais características. Além disso, verificou-se que não existem diferenças significativas para as diferentes regiões metropolitanas brasileiras e para os anos compreendidos no período considerado. Essa seção ainda analisou os efeitos de tais variáveis segundo a qualificação dos indivíduos e mostrou-se que, quanto mais qualificado, menor o efeito das variáveis de atributos pessoais na probabilidade de conseguir emprego ou manter-se empregado.

Apesar de as matrizes de transição oferecerem resultados importantes quanto ao objetivo do trabalho, não é possível através das mesmas fazer uma análise ceteris paribus das variáveis utilizadas controlando pelos atributos pessoais de cada indivíduo. Para isso, optamos por utilizar recursos econométricos e, através do modelo logit multinomial que se encontra na próxima seção, complementamos os resultados aqui encontrados.

\subsection{MODELO LOGIT}

A Tabela 1 traz os resultados do modelo geral e é dividida em duas colunas: desemprego - desemprego; desemprego - inativo. A primeira representa o resultado para indivíduos que mantiveram a condição de desempregado entre a primeira e a quinta entrevista. A segunda coluna representa o resultado para indivíduos que saíram da condição de desempregado na primeira entrevista para a condição de inativo na quinta. Na subdivisão dessas colunas, temos a coluna Coeficiente, que representa o valor estimado do coeficiente; o Erro Padrão, para fins de cálculo da significância do coeficiente; e a Razão de Chance, que, quando o coeficiente é positivo, representa a razão de chance da variável dependente assumir o valor um em relação à variável de referência.

A começar pela significância dos coeficientes estimados, ao nível de $5 \%$, todas as variáveis consideradas para a análise dos indivíduos que se mantiveram desempregados em um período de um ano foram estatisticamente significativas. Para os coeficientes referentes aos resultados para indivíduos que saíram do desemprego para a inatividade, apenas duas variáveis não foram estatisticamente significantes ao nível de $5 \%$ de confiança (2002 e Belo Horizonte). 
Tabela 1 - Resultado do modelo logit

\begin{tabular}{|c|c|c|c|c|c|c|}
\hline & \multicolumn{3}{|c|}{ Desemprego - Desemprego } & \multicolumn{3}{|c|}{ Desemprego - Inativo } \\
\hline & Coeficiente & Erro Padrão & & Coeficiente & Erro Padrão & $\begin{array}{c}\text { Razão de } \\
\text { Chance }\end{array}$ \\
\hline Chefe & $-0,398$ & 0,002 & 0,67 & $-0,503$ & 0,002 & 0,60 \\
\hline 2002 & 0,388 & 0,003 & 1,47 & $-0,001$ & 0,003 & 1,00 \\
\hline 2003 & 0,449 & 0,003 & 1,57 & $-0,050$ & 0,003 & 0,95 \\
\hline 2004 & 0,359 & 0,003 & 1,43 & 0,161 & 0,003 & 1,17 \\
\hline 2005 & 0,351 & 0,003 & 1,42 & $-0,019$ & 0,003 & 0,98 \\
\hline 2006 & 0,387 & 0,003 & 1,47 & 0,064 & 0,003 & 1,07 \\
\hline 2007 & 0,247 & 0,003 & 1,28 & $-0,079$ & 0,003 & 0,92 \\
\hline 2008 & 0,254 & 0,003 & 1,29 & 0,061 & 0,003 & 1,06 \\
\hline 2009 & 0,029 & 0,003 & 1,03 & $-0,158$ & 0,003 & 0,85 \\
\hline Homem & $-0,451$ & 0,001 & 0,64 & $-0,890$ & 0,001 & 0,41 \\
\hline Branco & $-0,128$ & 0,001 & 0,88 & 0,062 & 0,001 & 1,06 \\
\hline 30 a 49 anos & $-0,237$ & 0,001 & 0,79 & 0,028 & 0,001 & 1,03 \\
\hline 50 a 69 anos & $-0,019$ & 0,003 & 0,98 & 0,908 & 0,002 & 2,48 \\
\hline Escolaridade (4 a 10) & 0,076 & 0,003 & 1,08 & $-0,055$ & 0,002 & 0,95 \\
\hline Ecolaridade $(11+)$ & $-0,019$ & 0,003 & 0,98 & $-0,474$ & 0,003 & 0,62 \\
\hline Recife & 0,391 & 0,004 & 1,48 & 0,567 & 0,003 & 1,76 \\
\hline São Paulo & 0,450 & 0,003 & 1,57 & 0,040 & 0,002 & 1,04 \\
\hline Belo Horizonte & $-0,171$ & 0,003 & 0,84 & $-0,004$ & 0,003 & 1,00 \\
\hline Rio de Janeiro & 0,770 & 0,003 & 2,16 & 0,255 & 0,003 & 1,29 \\
\hline Salvador & 0,711 & 0,003 & 2,04 & 0,444 & 0,003 & 1,56 \\
\hline Intercepto & $-0,912$ & 0,005 & 0,40 & 0,008 & 0,004 & 1,01 \\
\hline
\end{tabular}

Fonte: Elaboração própria com base em dados de 2002 a 2011 da PME/IBGE.

Analisando inicialmente as variáveis que representam atributos individuais, os resultados do modelo logit geral mostram que, ceteris paribus, a probabilidade de um indivíduo chefe continuar desempregado é menor do que para os não chefes, resultado que corrobora a análise univariada realizada pela matriz de transição. Quanto à magnitude dessa diferença, pode-se dizer que a razão de chance de um indivíduo chefe encontrar emprego em relação ao não chefe é 1,49. As conclusões do trabalho de Cunha, Araújo e Lima (2011) estão em linha com esse resultado e mostram que, para os jovens, os chefes de família têm menor probabilidade de estarem desempregados. Tais autores argumentam que isso ocorre, pois, em geral, os chefes são responsáveis pela maior parte do orçamento doméstico, o que levaria sua busca por emprego a ser mais intensa. Essa tendência quanto à condição domiciliar também é apresentada em Sampaio (2012), alegando que as mulheres na condição de chefes de domicílio têm maior probabilidade de estarem empregadas do que as não chefes, mesma conclusão chegada por Mendonça, Lima, Lírio (2012) que justificam tal característica no fato de que mulheres jovens chefes tomam para si a responsabilidade de sustento da família. Quanto à condição de inatividade, a chance de um chefe passar de desempregado à 
condição de inativo também é menor do que a do não chefe, resultado também encontrado no trabalho de Fernandes e Picchetti (1999), que mostram que a menor taxa de inatividade ocorre para os chefes de família, seguidos por cônjuges e filhos.

Quanto ao sexo do indivíduo, observou-se que a razão de chance de um homem permanecer desempregado é menor do que a da mulher, como pode ser percebido através do sinal negativo do coeficiente da variável homem na Tabela 1. Além disso, pode-se dizer que ainda existe uma diferença expressiva na probabilidade de um homem encontrar ocupação em relação à mulher, já que a razão de chance nesse caso é de 1,57 . Tal resultado não é uma surpresa e é encontrado com unanimidade no estudo de vários outros autores como Cunha, Araújo e Lima (2011), Sampaio (2012), Fernandes e Picchetti (1999), Santos, Leal e Fernandes (2010) e Mendonça, Lima, Lírio (2012). Sabemos que tal tendência é resultado de, entre outros motivos, uma questão cultural que vem mudando nas últimas décadas. Quanto às mulheres jovens entre 15 e 24 anos, Mendonça, Lima, Lírio (2012) apontam para uma das principais causas do seu desemprego a gravidez precoce.

Ainda se tratando desse assunto, o relatório da International Labour Office (2012b) mostra que a diferença no desemprego entre homens e mulheres vinha diminuindo no mundo, tendo havido uma convergência na taxa de desemprego para os dois grupos entre os anos 2000 e 2007. De acordo com o estudo, apesar de que, em regiões como América Latina, as mulheres sempre terem tido maior taxa de desemprego do que os homens, em certas regiões do leste da Ásia, Europa Central e Ocidental, o gender gap chegou a ser negativo no período pré-crise, significando que o desemprego para as mulheres foi menor do que para os homens. Porém, o estudo mostra que a crise econômica parece ter piorado o gender gap no desemprego em todas as regiões, levando a zero o gender gap daquelas que o apresentaram negativo antes da crise.

Enquanto a análise das matrizes de transição mostra que a variável cor não gerou mudanças significativas na probabilidade de um indivíduo sair do desemprego para a situação de empregado, a análise controlada por mais variáveis revela que a variável é significativa e que brancos têm melhor chance de encontrar ocupação do que não brancos. Essa diferença, porém, não é tão grande como foi constatado para as variáveis sexo, condição domiciliar e idade, sendo a razão de chance de apenas 1,13 , isto é, o indivíduo branco tem apenas $13 \%$ a mais de chance de encontrar emprego do que o não branco. Apesar de outros estudos como Fernandes e Picchetti (1999), Fernandes, Lima e Santos (2008) e Cunha, Araújo e Lima (2011) mostrarem que a discriminação racial leva a uma diferença considerável no número de desempregados brancos e não brancos, os resultados geram conclusões otimistas em relação à diminuição da discriminação por cor no mercado de trabalho, pelo menos no que diz respeito à probabilidade de conseguir emprego em relação a se manter desempregado de um ano para o outro (análise dinâmica). 
A questão do desemprego para os jovens tem sido muito estudada e apresenta-se como um grande problema social para muitas economias. Como exemplos estrangeiros, podemos citar o cenário desfavorável no mercado de trabalho para os jovens de países como Espanha, Croácia, Eslováquia, Portugal, Grécia e Itália, o que mostra que a característica individual idade tem forte influência nas chances de conseguir emprego, principalmente em momentos de crise financeira. O relatório da International Labor Office (2012a) mostra que a crise econômica acabou abruptamente com o decrescimento da taxa de desemprego que vinha ocorrendo para os jovens entre 2000 e 2007, e discute as consequências da crise no mercado de trabalho para os jovens, sendo as principais o aumento da vulnerabilidade em relação a choques futuros e a instabilidade social gerada. Em relação ao Brasil, o cenário para os jovens não é diferente. A Tabela 1 mostra que a probabilidade de um indivíduo que tem entre 30 e 49 anos sair do desemprego para a condição de empregado é quase $26 \%$ (razão de chance de 1,26) maior do que a de um jovem que tem entre 15 e 29 anos. Até mesmo os indivíduos mais velhos (entre 50 a 69 anos) apresentaram uma leve vantagem na busca pelo emprego quando comparados aos jovens, com uma razão de chance de 1,01.

Muitos estudos foram realizados acerca da dificuldade de inserção do jovem no mercado de trabalho no Brasil e, dentre os principais motivos para tal ocorrência, destacam-se: a falta de experiência, o fato de muitos deles adiarem a entrada no mercado de trabalho para se qualificar ou, pelo lado dos empregadores, a dificuldade de avaliar a qualificação de um indivíduo que apresentou pouca ou nenhuma experiência no mercado de trabalho. Como exemplos de trabalhos de destaque nesse assunto, podemos citar Camargo e Reis (2005), que estudaram o desemprego para os jovens brasileiros através da perspectiva dos efeitos de estabilização da inflação; Cunha, Araújo e Lima (2011), que estudaram os determinantes do desemprego e inatividade dos jovens no Brasil metropolitano em 2007; Fernandes, Lima e Santos (2008), que pesquisaram a exclusão das mulheres jovens no Brasil; e Mendonça, Lima, Lírio (2012), que estudaram os determinantes da inserção de mulheres jovens no Nordeste.

Tendo em mente que um dos objetivos é identificar quais grupos de trabalhadores sofrem mais com o desemprego de acordo com sua qualificação, as variáveis de escolaridade inseridas no modelo logit multinominal apresentam importantes conclusões. A começar pelos indivíduos semiqualificados (4 a 10 anos de estudo) comparados aos menos qualificados ( 0 a 3 anos de estudo), temos que os semiqualificados apresentam maior probabilidade de manterem-se desempregados. De acordo com os resultados da Tabela 1 , pode-se dizer que a chance de um indivíduo semiqualificado continuar desempregado é $8 \%$ maior do que a de um indivíduo não qualificado (razão de chance de 1,08). Além disso, observa-se que os indivíduos mais qualificados são os que apresentam maior probabilidade de conseguir emprego. Tais 
resultados vão de encontro ao estudo realizado por Camargo e Reis (2005), que alegam que a curva de desemprego no Brasil em relação à qualificação do trabalho tem um formato de U-invertido, em que os trabalhadores de média qualificação são aqueles que apresentam maior desemprego. Os autores ainda sugerem que a justificativa para as piores condições de desemprego para os semiqualificados deriva-se do problema da assimetria da informação, já que um grupo tão heterogêneo como o que inclui indivíduos que têm entre 4 e 10 anos de estudo apresenta maior dificuldade para a avaliação feita pelos empregadores na decisão de contratação, levando-os a contratar menos trabalhadores desse grupo.

Quanto à região metropolitana, a análise ceteris paribus não apresentou diferenças significativas em relação à análise univariada realizada através das matrizes de transição. Dado que Porto Alegre foi a região metropolitana utilizada como base de referência, os resultados da Tabela 1 permitem concluir que os moradores de Belo Horizonte e Porto Alegre são os que têm maior probabilidade de encontrar emprego, enquanto indivíduos de Salvador são os que têm maior probabilidade de se manter desempregados. Porém, é importante ressaltar que, pelo menos para os indivíduos que mantiveram a condição de desemprego entre a primeira e a quinta entrevista, todas as dummies de Região Metropolitana mostraram-se significativas, o que sugere que localidade é uma variável que influencia a busca pelo emprego.

Por último, apresentamos os resultados para as dummies de tempo. Ao contrário das conclusões chegadas inicialmente através das matrizes de transição, o resultado do modelo multivariado aqui apresentado mostra que as variáveis dos anos entre $2001 \mathrm{e}$ 2010 são estatisticamente significantes. Com isso, não descartamos a hipótese de que as variáveis de ano exerçam influência na probabilidade de um indivíduo conseguir emprego ou manter-se desempregado no período considerado. Além disso, o resultado da análise de tais variáveis é nada menos do que surpreendente. Após já termos concluído anteriormente que a crise financeira não causou significativos problemas na taxa de desemprego no Brasil, a Tabela 1 mostra ainda que a probabilidade de indivíduos encontrarem emprego após a crise de 2008 cresceu. Por último, os resultados mostram que a chance de manter-se desempregado decresce de 2003 a 2010, um retrato da melhora do mercado de trabalho brasileiro ao longo da última década em relação ao desemprego.

Ao considerar os diferentes grupos de qualificação, foi possível perceber qual grupo mais se beneficiou da queda de desemprego no Brasil nos anos entre 2002 e 2011, bem como quais atributos pessoais apresentam maior influência na chance de os indivíduos encontrarem emprego. Um quadro comparativo com os coeficientes e razão de chance para os indivíduos que se mantiveram desempregados para os três grupos de qualidade de mão de obra é apresentado a seguir. 
Tabela 2 - Resultados do modelo logit para os três grupos de qualidade de mão de obra

\begin{tabular}{lcccccccc}
\hline & \multicolumn{3}{c}{ Coeficiente } & \multicolumn{3}{c}{ Razão de Chance } \\
\cline { 2 - 11 } & Geral & Não Qual. & $\begin{array}{c}\text { Semi } \\
\text { Qual. }\end{array}$ & $\begin{array}{c}\text { Qualifi- } \\
\text { cados }\end{array}$ & Geral & $\begin{array}{c}\text { Não } \\
\text { Qual. }\end{array}$ & $\begin{array}{c}\text { Semi } \\
\text { qual. }\end{array}$ & $\begin{array}{c}\text { Qualifi- } \\
\text { cados }\end{array}$ \\
\hline Chefe & $-0,98$ & $-0,505$ & $-0,515$ & $-0,224$ & 0,67 & 0,60 & 0,60 & 0,80 \\
2002 & 0,388 & 0,475 & 0,386 & 0,410 & 1,47 & 1,61 & 1,47 & 1,51 \\
2003 & 0,449 & 0,954 & 0,402 & 0,454 & 1,57 & 2,60 & 1,49 & 1,57 \\
2004 & 0,359 & 0,839 & 0,300 & 0,380 & 1,43 & 2,31 & 1,35 & 1,46 \\
2005 & 0,351 & 0,580 & 0,344 & 0,359 & 1,42 & 1,79 & 1,41 & 1,43 \\
2006 & 0,387 & 0,291 & 0,393 & 0,409 & 1,47 & 1,34 & 1,48 & 1,50 \\
2007 & 0,247 & 0,840 & 0,224 & 0,227 & 1,28 & 2,32 & 1,25 & 1,26 \\
2008 & 0,254 & 0,465 & 0,217 & 0,281 & 1,29 & 1,59 & 1,24 & 1,32 \\
2009 & 0,029 & 0,401 & $-0,052$ & 0,062 & 1,03 & 1,49 & 0,95 & 1,06 \\
Homem & $-0,451$ & $-0,508$ & $-0,538$ & $-0,387$ & 0,64 & 0,60 & 0,58 & 0,68 \\
Branco & $-0,128$ & $-0,190$ & $-0,204$ & $-0,072$ & 0,88 & 0,83 & 0,82 & 0,93 \\
30 a 49 anos & $-0,237$ & $-0,179$ & $-0,349$ & $-0,162$ & 0,79 & 0,84 & 0,71 & 0,85 \\
50 a 69 anos & $-0,019$ & 0,180 & $-0,205$ & 0,110 & 0,98 & 1,20 & 0,81 & 1,12 \\
Recife & 0,391 & 0,053 & 0,463 & 0,360 & 1,48 & 1,05 & 1,59 & 1,43 \\
São Paulo & 0,450 & 0,065 & 0,571 & 0,376 & 1,57 & 1,07 & 1,77 & 1,46 \\
Belo Horizonte & $-0,171$ & $-0,252$ & $-0,188$ & $-0,157$ & 0,84 & 0,78 & 0,83 & 0,85 \\
Rio de Janeiro & 0,770 & 0,337 & 0,783 & 0,784 & 2,16 & 1,40 & 2,19 & 2,19 \\
Salvador & 0,711 & 0,398 & 0,710 & 0,718 & 2,04 & 1,49 & 2,03 & 2,05 \\
Intercepto & $-0,912$ & $-0,39$ & 0,709 & $-1,024$ & 0,40 & 0,43 & 0,49 & 0,36 \\
\hline
\end{tabular}

Fonte: Elaboração própria com base em dados de 2002 a 2011 da PME/IBGE.

Ao nível de significância de 5\%, constatamos que todos os coeficientes encontrados para as variáveis independentes em relação à variável dependente desemp2 (manter-se desempregado em relação a obter ocupação) são estatisticamente significantes para todos os grupos.

A análise dos resultados das dummies temporais na Tabela 2 permite concluir não só que todos os grupos beneficiaram-se com a queda do desemprego, mas que o grupo dos indivíduos menos qualificados foi o que mais se beneficiou. A Tabela 8 mostra que a chance de um indivíduo não qualificado continuar desempregado em 2003 era 160\% maior do que em 2010, enquanto para os semiqualificados a chance era de apenas $49 \%$ maior e, para os mais qualificados, 57\%. Para os menos qualificados, essa diferença foi caindo ao longo dos anos, até que, em 2009, a chance dos desempregados que não conseguiram encontrar uma colocação foi de apenas 50\% a mais do que em 2010. Enquanto isso, a razão de chance de um indivíduo continuar desempregado para os grupos semiqualificados e mais qualificados não mudou tanto entre 2003 e 2010, apresentando um decaimento muito mais modesto do que o dos menos qualificados. Essa conclusão está em linha com o fato de que, hoje, no Brasil, empregadores encontram dificuldade para contratar mão de obra pouco qualificada. Dentre os motivos para a 
melhora significativa das chances de um indivíduo menos qualificado conseguir emprego nos últimos dez anos no Brasil foi a forte geração de empregos em ocupação com baixo nível de qualificação, como aponta Saboia et al. (2009).

Além disso, é possível concluir que os semiqualificados são aqueles que apresentam maior diferença na probabilidade de encontrar emprego devido aos atributos pessoais. Já os mais qualificados apresentaram os menores valores para razões de chance, o que significa que a discriminação baseada em atributos individuais é menor para esse grupo do que para os demais.

Em relação à condição domiciliar, observa-se que, independentemente da qualificação, ser chefe leva à maior probabilidade de conseguir emprego. Porém, essa variável é menos determinante na chance de tal ocorrência para os mais qualificados do que para os menos qualificados. A mesma linha de raciocínio é aplicável à variável homem. A chance de um homem qualificado conseguir emprego é $47 \%$ maior em relação à mulher qualificada, enquanto essa diferença sobe para $71 \%$ para os semiqualificados e $66 \%$ para os menos qualificados. Em relação à cor, a chance de um branco conseguir emprego é de apenas $8 \%$ a mais do que um não branco para os indivíduos mais qualificados, mas para os semiqualificados e menos qualificados, essa diferença chega a $23 \%$ e $21 \%$ respectivamente. Com relação à idade, os jovens semiqualificados parecem ser os que mais sofrem com discriminação quando comparados a indivíduos das demais faixas etárias. Os resultados mostram que a probabilidade de um indivíduo que tem entre 30 e 49 anos encontrar uma ocupação é $42 \%$ mais alta do que a de um jovem para o grupo dos semiqualificados, comparado a apenas $20 \%$ para os menos qualificados e $18 \%$ para os mais qualificados.

Quanto às regiões metropolitanas, a Tabela 2 mostra que as maiores diferenças nas razões de chance também ocorrem para os semiqualificados. Com destaque para os indivíduos residentes em São Paulo, Recife e Rio de Janeiro, é possível ver que a chance de uma pessoa semiqualificada dessas regiões comparadas a uma pessoa de Porto Alegre continuar desempregada é muito maior do que para os indivíduos mais qualificados ou menos qualificados.

Assim, podemos concluir que o grupo dos semiqualificados não só sofrem com a questão da assimetria da informação abordada por Camargo e Reis (2005), mas também se apresentam como os mais sensíveis à influência dos atributos individuais na probabilidade de sucesso no mercado de trabalho.

Em suma, os resultados do modelo Logit mostram que todos os grupos de qualificação de mão de obra ganharam com a queda do desemprego no Brasil na última década, sendo os menos qualificados aqueles que mais se beneficiaram. Esta sessão também mostrou que o grupo dos semiqualificados são os mais sensíveis aos efeitos dos atributos individuais já que, para esse grupo, ser mulher, jovem, não branco e/ou não 
chefe leva a uma piora significativa nas probabilidades de sucesso no mercado de trabalho. Além disso, os resultados mostram que os mais qualificados são aqueles que têm sua chance de conseguir emprego menos afetadas por atributos individuais.

Os resultados alcançados nesse artigo contribuem com importante avanço em relação à literatura existente, à medida que se baseia em uma análise longitudinal dos dados da PME, o que implica resultados mais detalhados da evolução da probabilidade de desemprego para os grupos apresentados. Enquanto a maior parte dos estudos existentes apresenta uma análise de corte transversal de dados originados da PNAD, apontando apenas para taxas de desemprego estáticas, o presente artigo permite interpretar o mercado de trabalho de forma dinâmica, discutindo probabilidades de transição entre as condições de emprego, desemprego e inatividade.

\section{CONCLUSÃO}

Este artigo buscou estudar o mercado de trabalho brasileiro tanto de um ponto de vista geral quanto através da perspectiva da qualificação da mão de obra para o período compreendido entre 2002 e 2011. Dentre os principais objetivos do estudo, destacaram-se a busca pelas variáveis que explicam as probabilidades de sucesso dos indivíduos na procura por emprego, a identificação dos atributos pessoais que afetam tais probabilidades dependendo do nível de qualificação do indivíduo e a investigação de quais grupos (mais qualificados, semiqualificados ou menos qualificados) mais se beneficiaram com a queda da taxa de desemprego no Brasil na primeira década do século XXI. Os resultados se derivam de uma análise dinâmica que permite interpretar as mudanças no mercado de trabalho com base nas probabilidades de um indivíduo transitar entre as condições de emprego, desemprego e inatividade, e representam importante contribuição ao avanço da literatura sobre o tema, já que a maior parte dos estudos existentes apresenta uma análise de corte transversal de dados originados da PNAD, apontando apenas para taxas de desemprego estáticas.

Como principais motivações para o estudo, destacaram-se a teoria da busca do emprego de Layard, Nickell e Jackman (1991) e a teoria do investimento em capital humano de Becker (1975), além do cenário de prosperidade do mercado de trabalho brasileiro no período analisado, principalmente nos anos subsequentes à crise econômica mundial, quando a maior parte do resto do mundo experimentou uma piora significativa nas condições de emprego. Em relação a essa disparidade, as análises dos dados do IBGE de taxa de desocupação e os resultados apresentados pelo estudo mostram que, enquanto países como Estados Unidos, Portugal, Grécia, Itália e mais severamente Espanha, Croácia e Eslováquia têm lutado contra dificuldades na geração de 
emprego e taxas elevadas de desemprego após a crise, no Brasil, a probabilidade de os indivíduos encontrarem ocupação chegou a subir nesse período. Quando analisados os grupos que mais ganharam com a melhora do cenário do mercado de trabalho na última década e durante a crise, os resultados apontaram que os menos qualificados (indivíduos com 0 a 3 anos de estudo) tiveram suas chances de encontrar emprego consideravelmente aumentadas entre 2003 e 2010, sendo os que mais se beneficiaram. Essa descoberta explica a falta de mão de obra disponível para profissões com baixo nível de qualificação no Brasil atualmente, o que pode ser um dos motivos do aumento do salário médio de tais trabalhadores.

Quanto às variáveis de atributo individual que mais influenciam as probabilidades de sucesso de um indivíduo na busca de ocupação, várias conclusões foram levantadas. Baseado nos dados da PME e através da análise de matrizes de transição, bem como nos resultados do modelo Logit Multinomial empregado, foi possível identificar que as variáveis sexo, condição no domicílio, idade e escolaridade têm grande influência nas chances de um indivíduo conseguir emprego. Concluiu-se que ser chefe no domicílio, ser homem, ter idade entre 30 e 49 anos, ou ter 11 ou mais anos de estudo, são características que aumentam a probabilidade de conseguir emprego ou manter-se empregado quando comparado às pessoas que não possuem tais características. Quanto aos resultados baseados nas regiões metropolitanas, o estudo mostrou que não existem diferenças significativas para as diferentes regiões consideradas, o que sugere que a variável de localidade não exerce forte influência na dinâmica do mercado de trabalho brasileiro.

O estudo das matrizes de transição, de acordo com a qualificação, mostrou que quanto maior é a escolaridade do indivíduo, menor o efeito das variáveis de atributos pessoais na probabilidade de conseguir emprego ou manter-se empregado. Em outras palavras, de acordo com as matrizes construídas, ter menos qualificação significa ter chances muito menores de conseguir emprego se o indivíduo for mulher, jovem ou não chefe. Nesse aspecto, o dado que mais chamou a atenção foi a diferença na chance de uma mulher conseguir emprego quando comparada a um homem, sendo esta $21 \%$ para indivíduos não qualificados e $14 \%$ para os mais qualificados.

Assim como identificado através das matrizes de transição, os resultados do modelo Logit também apontaram para o grupo dos mais qualificados como sendo aqueles em que atributos individuais menos interferem nas chances de conseguir emprego. Tais resultados sugerem que a melhor estratégia na busca pelo sucesso profissional para aqueles que possuem atributos pessoais menos favorecidos seja a busca por investimento em capital humano próprio e, ainda, que um maior esforço por parte do Estado na busca por maior escolarização dos brasileiros (principalmente jovens, mulheres e não brancos) pode resultar em menor desigualdade no mercado de trabalho no Brasil. 
Por fim, os resultados do modelo Logit permitiram concluir que o grupo dos semiqualificados são os mais sensíveis aos efeitos dos atributos individuais. Para esse grupo, pertencer a uma das classes de sexo, idade, cor ou condição domiciliar desfavorecidas leva a uma piora significativa nas probabilidades de sucesso no mercado de trabalho. Nesse sentido, os resultados do modelo mostraram que a chance de um homem semiqualificado conseguir emprego é $71 \%$ maior em relação à mulher semiqualificada, enquanto essa diferença cai para $66 \%$ para os menos qualificados e para $47 \%$ entre os qualificados. Em relação à cor, a diferença na chance de um branco comparado a um não branco no grupo de semiqualificados conseguir emprego é de $23 \%$, contra apenas $8 \%$ para o grupo dos qualificados. Para o quesito idade, os resultados mostraram que a probabilidade de um indivíduo que tem entre 30 e 49 anos encontrar uma ocupação é $42 \%$ mais alta do que a de um jovem para o grupo dos semiqualificados, comparado a apenas $20 \%$ para os menos qualificados e $18 \%$ para os mais qualificados.

\section{REFERÊNCIAS BIBLIOGRÁFICAS}

BECKER, S. G. Human capital: a theoretical and empirical analysis, with special reference to education. 2 ed. Nova York: Columbia University, 1975.

CAMARGO, J. M.; REIS, M. C.; Desemprego dos jovens no Brasil: os efeitos da estabilização da inflação em um mercado de trabalho com escassez de informação. Revista Brasileira de Economia, Rio de Janeiro, v. 61, n. 4, p. 493-518, out./dez. 2007.

CAMARGO, J. M.; REIS, M. C.; Desemprego: o custo da desinformação. Revista Brasileira de Economia, Rio de Janeiro, v. 59, n. 3, p. 381-42, jul./set. 2005.

CLARK, K. B.; SUMMERS, L. H. “Unemployment insurance and labor market transitions”. In: SUMMERS, L. H. Understanding unemployment. Cambridge: MIT, 1990.

CUNHA, D. A.; ARAÚJO, A. A.; LIMA, J. E.; Determinantes do desemprego e inatividade de jovens no Brasil metropolitano. Revista de Economia e Agronegócio, Viçosa, v. 9, n. 3, p. 369 392, set. 2011.

FERNANDES, R. O.; PICCHETTI, P. Uma análise da estrutura do desemprego e da inatividade no Brasil metropolitano; Pesq. Plan. Econ., Rio de Janeiro, v. 29, n. 1, p. 87-112, abr. 1999.

FERNANDES, R. A. S.; LIMA J. E.; SANTOS, C. M. A exclusão social de mulheres jovens, com idade entre 15 a 24 anos, no mercado de trabalho no Brasil. Revista de Economia e Administração, São Paulo, v. 7, n. 2, p. 125-136, abr./jun. 2008.

GUJARATI, D. N. Basic econometrics. Nova York: McGraw-Hill, 1995.

IPEA - INSTITUTO DE PESQUISA ECONÔMICA APLICADA. A desigualdade no desemprego no Brasil metropolitano. Comunicado da Presidência, n. 29. Brasília: IPEA, 2009. Disponível em: <http://www.ipea.gov.br/sites/000/2/comunicado_presidencia/09_09_22_ComunicaPresi_29_DesigualnoDesemprego.pdf>. Acesso em: 10 dez. 2012. 
ILO - INTERNATIONAL LABOR OFFICE, Global employment trends for youth 2012. Geneva: ILO, May 2012a. Disponível em: <http://www.ilo.org/wcmsp5/groups/public/---dgreports/--dcomm/documents/publication/wcms_180976.pdf>. Acesso em: 29 jan. 2013.

ILO - INTERNATIONAL LABOR OFFICE. Global employment trends for women. Geneva: ILO, Dec. 2012b. Disponível em: <http://www.ilo.org/wcmsp5/groups/public/---dgreports/--dcomm/documents/publication/wcms_195447.pdf>. Acesso em: 29 jan. 2013.

LAYARD, R.; NICKELL, S.; ACKMAN, R. Unemployment macroeconomic performance and the labour market. Oxford: Oxford University, 1991.

MENDONÇA, T. G.; LIMA, J. R.; LÍRIO, V. S. Determinantes da inserção de mulheres jovens no mercado de trabalho nordestino. Revista Econômica do Nordeste, Fortaleza, v. 43, n. 4, 2012.

POCHMANN, M. et al. Demanda e perfil dos trabalhadores formais no Brasil em 2007, Brasília: IPEA, nov. 2007. Mimeo.

RIBAS, R. P.; SOARES, S. S. D. Sobre o painel de pesquisa mensal de emprego (PME) do IBGE. Texto para Discussão, IPEA, Rio de Janeiro, n. 1348, ago. 2008.

SABOIA, J. (Coord.). Tendências da qualificação da força de trabalho. Rio de Janeiro: UFRJ, 2009. (Estudo transversal, 04). Relatório final do Estudo Transversal "Qualificação da Força do Trabalho". Projeto de Pesquisa "Perspectivas do Investimento no Brasil”. Rio de Janeiro: Instituto IE-UFRJ / Campinas: IE-UNICAMP, 2008/2009.

SAMPAIO, A. V. Estrutura do mercado de trabalho metropolitano na Região Sul do Brasil, em 1995 e em 2005. Nova Economia, Belo Horizonte, v. 22, n. 1, p. 85-115, jan./abr. 2012.

SANTOS, C. M. S; LEAL F. D.; FERNANDES, R. A. S. A exclusão social de mulheres jovens, com idade entre 15 a 24 anos, no mercado de trabalho mineiro. In: SEMINARIO SOBRE A ECONOMIA MINEIRA, 14., 2010, Diamantina. Anais... Belo Horizonte: UFMG/CEDEPLAR, 2010.

WOOLDRIDGE, J. M. Introdução à econometria: uma abordagem moderna. São Paulo: Thomson Learning, 2007. 